\title{
A Survey of IEEE 802.11 Protocols: Comparison and Prospective
}

\section{Dong Chen ${ }^{1, a, *}$}

${ }^{1}$ School of Electronics and Information, Northwestern Polytechnical University, Xi'an, Shaanxi, China

a chendongcharlie@mail.nwpu.edu.cn

*corresponding author

Keywords: Wireless network, 802.11

\begin{abstract}
Since the base version of IEEE 802.11 released in 1997, wireless local area network (WLAN) has been developing rapidly, and it has gone through many versions and amendments. The 802.11 protocol is now very sophisticated through 20 years of modification. Therefore, it is very hard to understand all improvements, which confuses researchers sometimes, making it not easy to discover the trend of development. This paper tries to summarize the key technologies that pushed WLAN forward among all important amendments, and then lists the latest research focus for the next generation of 802.11 family. The paper would provide assist researchers to find their field of interest.
\end{abstract}

\section{Introduction}

Beginning from 1997, IEEE 802.11 protocol (or protocol family after the release of IEEE 802.11a/b in 1999) is dedicated for a better quality of wireless local area network(WLAN) through establishment and amendment of WLAN standards on media access control (MAC) and physical layer (PHY) specifications. The base version, 802.11, was released in 1997, and then many amendments were released. Today this process of update has not finished yet. As the author writing this report, a new version of amendment, 802.11ax led by Dr. Osama Aboul-Magd is just about to be approved, and the next version, 802.11ay, is under development and discussion.

Through 20 years of modification, the 802.11 protocol is now very sophisticated and hard to understand (In fact, the original version has 459 pages, but the 2012 version 2,793 pages with 23 annexes). In this report, we will try to analyze these important amendment, and explore the reason why the QoS (or Quality of experience, i.e., QoE) could be improved through these updates through comparison. This paper will also predict the future (unprofessionally) of 802.11 protocol family based on my own reading and knowledge.

The rest of the paper is organized as follows. From Section 2 to 5 , we describe the basic specifications of previous versions of 802.11 family as well as their core technologies. In Section 6, a detailed introduction of 802.11 ax will be provided, including its usages and proposed improvements. In Section 7, we will look into other research focuses, such as higher-frequency communication and machine learning, and provide trend of future development of WLAN.

\section{IEEE 802.11 Base Version}

The base version, or so-called IEEE 802.11-1997, as this paper mentioned above, was released in 1997. At the very beginning of 802.11, this protocol was developed for rapid deployment of a wireless portable network. That is to say, the first priority is mobility rather than network speed. Though this version was no longer being used, the fundamentals were well defined in it, therefore it is interesting to look into it.

\subsection{MAC Architecture and Key Technologies}

In Medium Access Control (MAC) sublayer, there were 2 functions included: the distributed coordination function (DCF) and the point coordination function(PCF). 


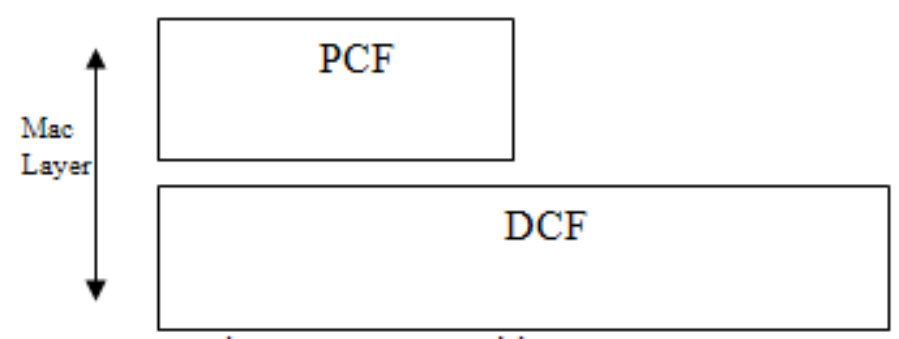

Figure 1 MAC architecture

The PCF is required for contention free services, while the DCF is used for contention services and basis for PCF. The architecture was well defined and it is still being used in latest version (with updates, of course). Figure 1 shows the original architecture of MAC sublayer.

\subsubsection{Distributed Coordination Function}

DCF, known as carrier sense multiple access with collision avoidance (CSMA/CA), plays an essential role in IEEE 802.11 family. It allows for automatic sharing of public resources among all stations (STAs). In most cases, the channels STAs working on is busy and thus contention is somehow inevitable. In this situation, when an STA desires to initial a frame exchange, it shall follow a series of basic operations. The STA shall listen to the channel and wait until it determines that the medium would be idle for longer than a distributed inter-frame spacing (DIFS) period. Then, the random backoff time shall be applied given the equation:

\section{Backoff Time $=$ Random ()$\times$ aSlotTime}

Here, Random() generates a Pseudorandom integer which satisfy a uniform distribution over the interval [0, CW], where $\mathrm{CW}$ is related to the count of retries, and aSlotTime an attribute dependent on medium. The whole backoff procedure is briefly introduced in 2.1.2.

\subsubsection{Backoff procedure}

A backoff procedure shall be invoked if either the physical or virtual carrier sense mechanism determine the channels are busy, or transmitting STA indicates a failed transmission.

To begin the backoff procedure, the STA shall set its backoff timer with the formula in 2.1.1, and all timers shall occur following a DIFS period. When the timer occurs, STA would determine whether the channel is idle for every slot time. If the channel is idle, timer would decrease slot time, otherwise the timer would suspend until the channel is idle again (and wait for another DIFS period). The transmission shall commence when timer reaches 0. Figure 2 shows the whole backoff procedure.

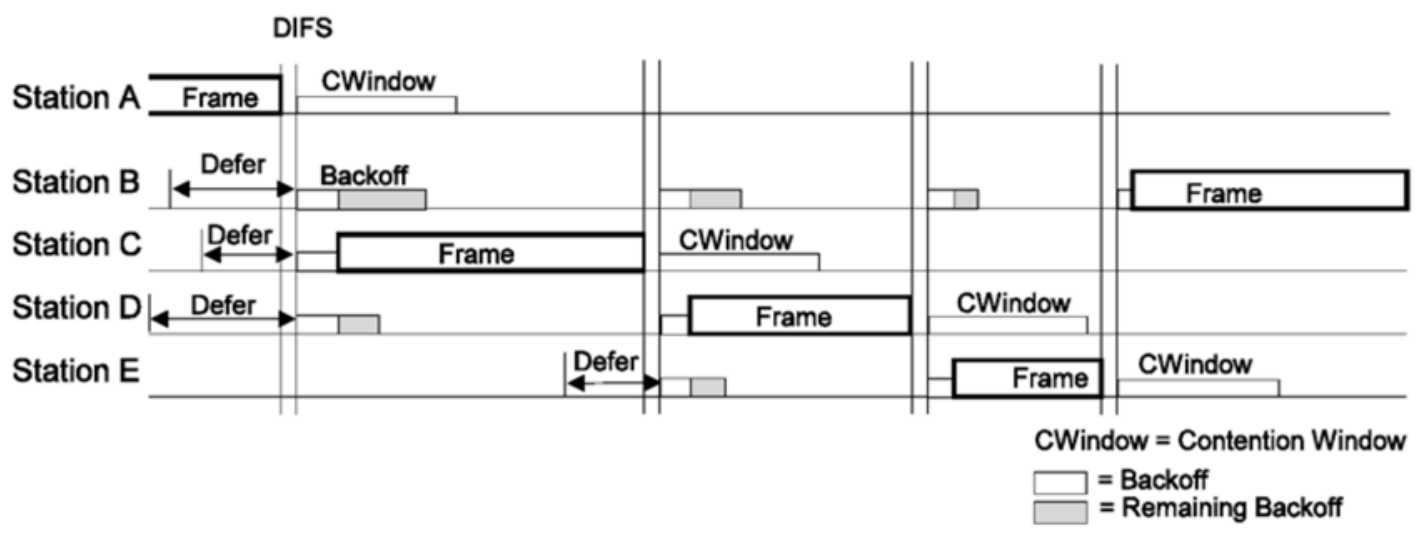

Figure 2 Backoff Procedure [1]

The effect of backoff procedure is that STA with least Random() would win the channel if it is being contended by multiple STAs. The backoff procedure is very basic but effective, and it continues to work in later versions with very little updates. 


\subsubsection{Virtual Carrier Sense Mechanism}

The virtual carrier sense mechanism is achieved by distributing reservation information announcing the impending use of the medium. A common method is the exchange of Request to Send (RTS) and Clear to Send (CTS) frame before the data being transmitted. Any STAs that receive the RTS and CTS towards other STA will save the information in Network allocation vector (NAV). NAV will determine the channel condition and it would be used as the result of virtual carrier sense mechanism. This mechanism handled well the problem of hidden nodes and thus promoting the efficiency of system. However, RTS/CTS are not mandatory frames. In a contentionfree environment, or if data packet is very small, such an operation of channel reservation would decrease the efficiency of transmission.

\subsection{PHY Specifications and Key Technologies}

In original version of 802.11, the protocol utilizes the microwave ISM band at $2.4 \mathrm{GHz}$ with 3 different physical layer specifications: frequency hopping (FH PHY), direct sequence spread spectrum (DSSS PHY) and infrared light (IR PHY).

IR PHY, though with multiple unique advantages, was not widely used due to security problems and relatively short range.

\subsubsection{Physical Layer Overview}

PHY layer is divided into 2 sublayers: The Physical Layer Convergence Procedure (PLCP) sublayer and the Physical Medium Dependent (PMD) sublayer. PLCP is the sublayer between MAC and actual transmission, i.e., PMD layer, which receives data from MAC and adds its header, then pass it to PMD, while PMD sublayer takes responsibility for transmitting.

802.11 uses 2.4GHz ISM band, requiring no licensing as long as the transmitting power and radiation satisfy regulations. However, because of it, all STAs must work under possible noise and contentions.

\subsubsection{FH Transmission}

FH PHY implements rapid changes on the transmission frequency in a predetermined pseudorandom pattern. Available frequency band is divided into a series of frequency slot with maximum width of $1 \mathrm{MHz}$, and the time is divided likewise. When transmitting, transmitter will use the pattern to decide the frequency slot for transmission for every time slot. Only if the transmitter and the receiver are using the same channel can the transmission succeed. There may exist multiple transmitters and receivers, and therefore it is not enough if only one patter exists. There are over 20 hop sets, in different region which may differ but are orthogonal, i.e., these sequences would not overlap. When a STA is joining any FY network, it has to synchronize with the network, the specifications for which are: region, sequence number and hop index.

FH PHY utilizes Gaussian frequency shift keying (GFSK). It encodes data in a series of frequency changes in the carrier. One advantage of using GFSK is that GFSK is relatively immune to noise, since noise usually change the amplitude of a signal. The simplest GFSK is 2-level GFSK, in which frequency has only 2 possibilities. But in this way the symbol rate is relatively low. An improved way is 4-level GFSK, doubling the symbol rate but also the system complexity.

\subsubsection{Direct Sequence Spread Spectrum Transmission}

In initial 802.11 specifications, a physical layer based on low-speed, direct-sequence spectrum was introduced. Compared with FH PHY, DSSS PHY costs more power, but this high cost comes with a benefit of much higher data rates.

Direct-sequence transmission is a spread-spectrum technique to transmit a signal over a much wider frequency band. The basic approach is to spread the signal by changing the carrier across a wide band, while receiver shall perform a correlation operation to look for the changes. When transmitting data, direct-sequence-modulated works by applying a chipping sequence to the data stream. Direct-sequence-modulated signals could deal better with interference than frequency- 
hopping-modulated signals because this correlation process enables DS systems to work around narrowband interference more effectively.

DSSS uses differential phase shift keying (DPSK) as the basis. It encodes data in phase changes in the transmitted signal. Similarly, the simplest DPSK is differential binary phase shift keying (DBPSK), encoding only 0 and 1: reference wave to be 0 while wave with a phase shift of $\pi$ to be 1 . Advanced transmitters and receivers could encode multiple bits per symbol by implementing DQPSK, which encodes 2 bits in 1 symbol.

\section{802.11a}

802.11a was released in 1999. 802.11a is an amendment at a much higher transmission rate, but it was somehow unable to hit the market. Most devices currently do not support this standard of transmission, but it still provides a fundamental of transmission on higher-frequency band, and its later version, such as $802.11 \mathrm{n}$ that works on both bands, is very popular, being the mainstream standard of WLAN commercial devices.

\subsection{Specifications}

802.11a used same core protocols in the original version, but it operates on $5 \mathrm{GHz}$ band, which increases the maximum raw data rate of 54Mbps. The key improvement of 802.11 is the implementation of Orthogonal frequency-division multiplexing (OFDM).

\subsection{OFDM}

The base of OFDM is plain old frequency division multiplexing (FDM). FDM divides frequency band into slices, and there will be a gap between adjacent slices called guard band. When transmitting, different users would be allocated a different carrier, and they are permitted to transmit data on that band. FDM is widely used in the first-generation mobile communication. The advantage is that it increases the throughput by using subcarriers in parallel. But it also wastes the band because of guard bands.

OFDM is a solution to increase the throughput a step further than FDM by eliminating this guard band. In order to do so, OFDM resolves the problem of overlapping band from elimination, by carefully designing the subcarriers in such a way that at the center of each band, energy of other bands would be little and thus the signal could be distinguished. In this way, it is fine even the bands overlap, and in application there could be large number of closely spaced orthogonal subcarriers used to carry data.

Apparently, OFDM possesses a higher spectral efficiency than FDM. Other advantages include robustness against narrow-band interference and inter-symbol interference. It could be carried out with fast Fourier transform (FFT), which means the calculation could be minimized. However, it is sensitive to Doppler effect, i.e., the change in frequency or wavelength of a wave for an observer moving relative to the source, and frequency synchronization problems. The efficiency of OFDM may also be interfered by cyclic prefix and guard interval.

\section{IEEE 802.11b/g}

In the same year with 802.11a, 802.11b was released. But unlike 802.11a, 802.11b is a version widely used for over 10 years. It is still available on many devices. However, technically $802.11 \mathrm{~b}$ had little change on 802.11 base version. It focused on defining specifications and standards that govern transmission method.

IEEE 802.11g, released 4 years later, is an amendment mainly for 802.11b. It helps improve the data rate to $54 \mathrm{Mbps}$, same as $802.11 \mathrm{a}$, at $2.4 \mathrm{GHz}$ band. The reason why $802.11 \mathrm{~b}$ is still supported may be that $802.11 \mathrm{~g}$ shares many properties with 802.11b. (But this does not suggest devices with different protocol could work together) 


\subsection{Specifications}

802.11b define a maximum raw data rate of $11 \mathrm{Mbps}$ operating at $2.4 \mathrm{GHz}$ band. It kept using CSMA/CA as its media access method.

802.11g define a maximum raw data rate of $54 \mathrm{Mbps}$ operating at $2.4 \mathrm{GHz}$ band.

\subsection{Complementary code keying}

Complementary code keying (CCK) is a modulation method defined in $802.11 \mathrm{~b}$, considered to be a new modulation scheme. It encodes data in phase change like DPSK, but DPSK is such a scheme that the data and the symbol has a one-to-one correspondence, while CCK not. In 802.11b, it encodes 4 or 8 bits in 8 chips of symbols, given that:

$$
\begin{aligned}
& \mathbf{c}=\left(c_{0}, \ldots, c_{7}\right) \\
& =\left(\mathrm{c}^{\mathrm{j}\left(\dot{\phi}_{1}+\dot{\phi}_{2}-\dot{\phi}_{3}+\dot{\phi}_{4}\right)}, \mathrm{c}^{\mathrm{j}\left(\dot{\varphi}_{1}+\dot{\phi}_{2}-\phi_{4}\right)}, \mathrm{c}^{-\mathrm{j}\left(\dot{\phi}_{1}+\dot{\phi}_{4}\right)}, \mathrm{c}^{\mathrm{j}\left(\dot{\phi}_{1}+\dot{\phi}_{2}+\dot{\phi}_{3}+\dot{\phi}_{4}\right)}, \mathrm{c}^{\mathrm{j}\left(\dot{\phi}_{1}+\dot{\phi}_{2}+\dot{\phi}_{3}+\dot{\phi}_{4}\right)}, \mathrm{c}^{\mathrm{j}\left(\dot{\phi}_{1}+\dot{\phi}_{3}\right)}, \mathrm{c}^{\mathrm{j}\left(\dot{\phi}_{1}+\dot{\phi}_{2}\right)}, \mathrm{c}^{\mathrm{j}\left(\hat{\phi}_{1}\right)}\right)
\end{aligned}
$$

where $\mathbf{c}$ is the code word, and $\phi_{1}, \phi_{3}, \phi_{3}, \phi_{4}$ are determined by data. This is a form of the generalized Hadamard transform encoding, where $\phi_{1}$ is added to all code chips, $\phi_{2}$ is added to all odd code chips, $\phi_{3}$ is added to all odd pairs of code chips, and $\phi_{4}$ is added to all odd quads of code chips

Basically, CCK has an advantage of higher resistance of noise and multipath interference compared with QPSK, and is compatible to work with 1Mbps and 2Mbps WLAN. Therefore, CCK was finally applied as the modulation scheme in $802.11 \mathrm{~b}$, other candidates including OFDM, OCDM and MOK. Note that OFDM, though not accepted in 802.11b, was later applied in 802.11a in a $5 \mathrm{GHz}$ band.

\subsection{Improvement of $802.11 \mathrm{~g}$}

802.11g possesses a data rate of $54 \mathrm{Mbps}$, almost 5 times of the data rate 802.11b. It is largely because $802.11 \mathrm{~g}$ implements OFDM at $2.4 \mathrm{GHz}$ band, and this parallel usage of band greatly improve the spectral efficiency.

The security is another improvement in 802.11g. 802.11b only supports MAC filtering and SSID hiding. The authentication process only supports wired equivalent privacy (WEP). WEP was not a secure method of encryption due to a few major flaws of design discovered: over more and more tests by users, it was discovered that the key used for encryption can be reconstructed by listening on the network and catching packets. Therefore, many groups have found the way to compromise this way of encryption. Therefore, Wi-Fi Protected Access (WPA), and its higher version, WPA2, is to replace WEP. Compared with WEP, WPA and WPA2 requires more work for encryption. But this work worth it-the expertise required to break WPA and WPA2 is much higher, making the decipherment less valuable.

\section{5. $802.11 n / a c$}

IEEE 802.11n, the most popular version commercially, was first released in May 2009. After the problem of security being resolved and many new technologies developed at the physical layer, a much higher data rate was expected in the version, as well as the version after it.

IEEE 802.11ac is a version based on 802.11a and $n$ and further advancing the data rate of the WLAN. Basically, it increases most parameters related to the bit rate in order to make the transmission faster.

\subsection{Specifications}

802.11n works on both $2.4 \mathrm{GHz}$ band and $5 \mathrm{GHz}$ band, and the raw data rate is estimated to be up to $600 \mathrm{Mbps}$ (300 Mbps in practice), that is approximately 6 times larger than $802.11 \mathrm{~g}, 54 \mathrm{Mbps}$.

Unlike $802.11 \mathrm{n}, 802.11$ ac works only on $5 \mathrm{GHz}$ band, reaching a raw data rate of at least $1 \mathrm{Gbps}$, or at least $500 \mathrm{Mbps}$ for a single-link throughput. 


\subsection{Smart Antenna [5]}

Smart antennas, also known as adaptive array antennas or multiple antennas, are antenna arrays with smart signal processing algorithm used to identify signals as the direction of arrival.

Smart Antennas implements Spatial Division Multiple Access (SDMA), a method of multiplexing different from FDM or TDM, which uses the differences between signals traversing in different directions to distinguish each other. Compared with FDM or TDM, SDMA could decrease the inference of Rayleigh Scattering and Multipath interferences, can identify signals even in the same carrier or time slot. SDMA is such a multiplexing that can be used with others, and thus the spectral efficiency can be greatly improved. More details on this "combination" will be discussed in the next section.

The fundamental of smart antenna is Beamforming. The idea of beamforming is that the transmitter will obtain the information between itself and the receivers, such as the relative position and direction of receiver regard of itself and the properties of the channel. With all these information, the transmitter could adjust the all these parameters accordingly so that the signal power could be more focused on the direction of receivers, promoting the signal-to-noise ratio (SNR), and therefore the throughput.

Smart antennas use the core idea of beamforming and make a progress to it. The principle of smart antennas is to produces space directional beam with DSP techniques and the main beam is very focused on the direction of the receiver so that the SNR could be further promoted. Furthermore, smart antennas also use the differences we mentioned earlier of each station to transmit multiple signals on it array without interfering each other, again greatly increasing the efficiency.

\subsection{MIMO-OFDM}

In section 5.2 we mentioned that it is possible and prospective to combine several multiplexing to promote the data rate and spectral efficiency, Multiple-Input Multiple-Output OFDM (MIMOOFDM) is a good example of which. By implementing the technology of smart antennas, a station could transmit (or receive) to (or from) several stations at the same time, if all the conditions meet. Therefore, a Multiple-Input Multiple-Output system, known as MIMO was proposed and finally realized on 802.11n. And since MIMO is using spatial division, it is still possible to use other ways of division. Therefore, MIMO-OFDM was proposed in $802.11 \mathrm{n}$, and now is has become the foundation for most advanced WLANs.

\subsection{Improvement of 802.11ac}

As mentioned earlier, 802.11ac increased many parameters for a better bit rate (with the development of technology, of course). It works only at $5 \mathrm{GHz}$ band so that it could use a wider range of frequency. It implemented a higher-density modulation up to 256QAM, or a non-standard 1024QAM which further increases data rate at 25\%. It also used extended channel binding and more MIMO spatial streams.

\subsubsection{Binary Convolutional Code [6]}

It was written in the standard of 802.11ac that a different way of coding, binary convolutional coding (BCC), was required for all devices supporting 802.11ac, while in 802.11n it was Space-time block coding (STBC). STBC operates by transmitting multiple copies at different time on different antenna so that we can increase the reliability of transmission. BCC, quite different from STBL, is a kind of error-correcting code that generates parity symbols via the sliding application of a Boolean polynomial function to a data stream.

The difference between 2 coding above is their principles. As for STBL, the information bits are followed by their parity bits, while in convolutional codes we spread the information alone a sequence. This means that block code maps all the information in the block, but convolutional codes on code bits. BCC is a very efficient coding (but less efficient than turbo code that came later). BCC also possesses a better ability for error detect because the information is not only related 
to the bit received at one moment but the bits received earlier or the following ones. And thus the Rate of error falls exponentially.

\section{802.11ax}

802.11ax is the latest approved version, first proposed and led by Huawei. The scope of 802.11ax is to work on the following points:

1. Improve spectrum efficiency and area throughput;

2. Improve system and throughput in dense deployment;

3. Improve real world performance.

According to Huawei, the top data rate could reach $10 \mathrm{Gbps}$. It works on both $2.4 \mathrm{GHz}$ and $5 \mathrm{GHz}$ bands. The commercial products are estimated to hit the market in 2020.

\subsection{Intended Use Scenarios}

IEEE 802.11ax is facing an environment that is very different from that when first version of 802.11 was released. It is expected to cope with a more complex environment as more devices are connected and many other devices are sharing ISM band. Furthermore, users are using wireless network for multiple purposes, and thus higher throughput and smaller delay is preferred. Specifically, 802.11ax is expected to be optimized for the following environments. [9]

(1) Indoors environment. In an apartment building or enterprises, multiple APs may be set in a small area. This would lead to an increased interference due to dense deployment and distributed management.

(2) Outdoors environment. In this scenario, APs operate like base stations in cellular networks that covers a large number of devices. The main source of interference comes from all non-AP devices as they all need to connect to AP of this infrastructure BSS.

(3) Public WLAN access. In many public spaces such as airports or shopping malls, user density is expected to be high in business hours, which demands the APs to operate effectively in order to maintain the QoE by reusing spatial resources and coordinating users wisely.

\subsection{Orthogonal Frequency-Division Multiple Access [7]}

The main improvement of 802.11ax is the implementation of Orthogonal Frequency-Division Multiple Access (OFDMA), an advanced version of OFDM. It advanced OFDM by introducing multiple users in its modulation scheme.

Exploiting OFDMA into wireless networks has many advantages. First of all, it allows

multiple users simultaneously, so that QoS for each user could be promoted. Second, it is expected to achieve shorter delays and constant delays. Third, it enables a more flexible network because it enables single-frequency network coverage and offers frequency diversity. Moreover, it further improves OFDM robustness to fading and interference. [10]

There are certain disadvantages of OFDMA compared with OFDM. The most challenging problem is its higher sensitivity to frequency offsets and phase noise. Researchers are working hard to solve these problems so that 802.11ax could achieve better performance. [10]

\subsection{MAC-layer Enhancements}

\subsubsection{Spatial Reuse}

CSMA/CA is considered the core technique of 802.11 family because it provides a wise way to avoid collisions. However, as more devices connected to a single BSS, the area throughput actual decreases. TG ax purposed to use an alternative by setting energy level dynamically so that spatial resources can be reused. In practice, energy level is expected to be set lower so that the area is covers would be smaller than that of carrier sense.[11] In this way, multiple nodes may be connected even in a small area. The drawback of this approach is obvious: SNR decreases when we reduce the transmission power and this may lead to higher BER and higher chance to have hidden node problems. 


\subsubsection{New MAC-layer protocols}

As collisions in MAC-layer waste a lot of valuable resource, a possible enhancement in MAClayer is to redefine the MAC-layer protocol to prevent collision, i.e., collision-free protocols. Paper [8] presented 2 possibilities. The first is to move to a centralized solution, but as Hybrid coordination function Controller Channel Access (HCCA) never implemented in 802.11, this solution is very unlikely to realize. The second solution is to improve current CSMA/CA protocol: CSMA/ECA (CSMA with Enhanced Collision Avoidance). Paper [10] gives detailed information about this new purposed protocol.

\section{Prospect: Future of WLAN}

It is exciting to see that new technologies are being used in the development of WLAN, and now the amendment of 802.11 family are coming like "annual anniversary". In fact, the next version of 802.11, 802.11ay (still led by Huawei and Intel) has just finished its 0.3 version and the final version is estimated to complete 3 months later. In this chapter, we will first take a glance of this very new version, and discuss more on future development.

\subsection{11ay: Even Higher Frequency}

So far, there are only two bands in our discussion: 2.4GHz band and 5GHz band. Unfortunately, these two bands are very crowded, and narrow-not suitable for communication at an even higher speed. Especially 2.4GHz band, which is in the range of ISM band, shares the band even with microwave oven. Thus, an idea of using even higher frequency band came out. It was written in 802.11ad, but not much attention it acquired, though it did achieve maximum data rate of 7 Gbps. This next version, 802.11ay, focus again on the enhancement of throughput for Operation in License-Exempt Bands above $45 \mathrm{GHz}$.

In order to realize the target of supporting a maximum throughput of at least 20 gigabits per second at MAC layer, there are a few new technologies proposed to be used in this version. In fact, operating on $60 \mathrm{GHz}$ band, i.e., mmWave, is a new technology. Researcher have worked hard on this new model of transmission.

Another essential update is MU-MIMO, just like OFDMA to OFDM, to MIMO. In a similar way that OFDMA adds multiple access (multi-user) capabilities to OFDM, MU-MIMO adds multiple access (multi-user) capabilities to MIMO. It is important to note that MU-MIMO was also proposed in 802.11ad.

\subsection{Full Duplex}

So far, our discussion is limited to a fact that a station could transmit and receive data, but not simultaneously. However, this situation is about to change, soon. Related researches have been carried on for years and there are many available solutions proposed. Intuitively, by allowing stations to transmit and receive beams at the same time, the throughput could be doubled. Inspiringly, the figure is more than a double.

According to work by Jinnyeong Lee et al [12], this improvement is more than $100 \%$. They are also working on implementing Massive MIMO along with full duplex, and their simulation shows this could improve nearly $130 \%$ than using full duplex only, not to mention conventional half duplex.

In this stage, it is reasonable to assume that only access points possess full duplex, and based on this assumption Qiao Qu et al have proposed a method named FuPlex [13] that could still increase the throughput significantly, even in dense deployment.

\subsection{Machine Learning: an intelligent future}

Machine learning (ML), along with artificial intelligence (AI) and deep learning, is very popular in recent years in a wide range of areas. However, when it comes to network architecture, or more related to our course, WLAN, is not as heated as in other areas. Many researchers working on using AI or ML into network complainted that the modelling is too complex, hence many researches are 
not practical enough. There is also a voice that when designing network with ML, it seems that the designers are judging the system at the top level, i.e., they know everything, while none of the STAs, no matter it is a router or a switchboard, cannot have a full map of network.

Though the work is tough, there are many achievements so far. A common use of ML is recognition. With pre-trained system, such as neural networks and support vector machine(SVM), a ML approach could predict patterns that we are interested in. Mayank Agarwal et al. have developed an ML approach to detect De-Authentication DoS attacks in Wi-Fi networks. [14] They purposed an ML based Intrusion Detection System (IDS) to detect such scenarios. In this system, they implemented multiple ML based classifiers to enable a better performance and their experiments showed an accuracy exceeding 96\%. Oscar Puñal et al. presented an ML-based jamming detection for WLAN [15], using multiple metrics, including channel metrics, performance metrics ad signal metrics to collect data and multiple ML algorithm to recognize if there exists a jamming. Their research showed that supervised approaches exhibit similar performance while unsupervised learning ones is not suited for jamming detection.

In recently years, there are many on-line controllers for WLAN using machine learning algorithm. Chiapin Wang et al. purposed to use BP neural networks (NN) for management of WLAN resource allocation. [16] By implementing NN, the system is able to regulate itself accordingly and dynamically so that utility can be maximized. Pochiang Lin et al. tackle the optimization of frame-size using a ML-based adaptive approach. [17] The model collects data of throughput from all STAs and then abstract gradient information from the neural network they build such that frame size can be adjusted accordingly. Their simulation shows that the model they purposed outperforms the existing solutions.

\section{Conclusion}

From our research, it is obvious that wireless network has been a hot research topic during almost 20 years and it is still a focus in the near future due to the continuous increase of users' demand on wireless network. However, different from the base version of 802.11 protocols, latest versions of WLAN have some certain new features. In our paper, we find that instead of improving data rate at the PHY layer, recent researches focus on MAC layer [20], trying to reduce the overhead so that overall efficiency can be promoted. It is also a trend, like many other industries, to implement machine learning tools into communication, and thus computation ability of an AP would become more and more important in the future.

\section{References}

[1] IEEE. (1997). Part 11: Wireless LAN Medium Access Control (MAC) and Physical Layer (PHY) specifications. New York, USA.

[2] Stephens, A. (2017). IEEE 802.11(TM) WIRELESS LOCAL AREA NETWORKS. Retrieved from http://grouper.ieee.org/groups/802/11/

[3] Gast, M. S. L., Mike. (2002). 802. 11 Wireless Networks, The Definitive Guide. Oreilly, 32(11), 111-124.

[4] Liu, Z., Chen, Z., Wu, C., \& Wu, Z. (2005). Performance Simulation of Complementary Code Keying. Journal of Civil Aviation University of China, 23(z1), 79-81.

[5] Chandran, S. (2009). Smart Antennas for Wireless Communications (with MATLAB) (Gross, F.; 2005) [Reviews and Abstracts]. IEEE Antennas \& Propagation Magazine, 51(3), 134-134.

[6] Miller, F. P., Vandome, A. F., Mcbrewster, J., Federal, \& United. (2010). Federal Standard 1037C.

[7] Morelli, M., Kuo, C. C. J., \& Pun, M. O. (2007). Synchronization Techniques for Orthogonal Frequency Division Multiple Access (OFDMA): A Tutorial Review. Proceedings of the IEEE, 95(7), 1394-1427.

[8] Bellalta, B. (2015). Ieee 802.11ax: high-efficiency wlans. IEEE Wireless Communications, 23(1), 38-46. 
[9] Afaqui, M. S., Garcia-Villegas, E., \& Lopez-Aguilera, E. (2016). IEEE 802.11ax: challenges and requirements for future high efficiency wifi. IEEE Wireless Communications, PP(99), 2-9.

[10] Sanabria-Russo, L., Faridi, A., Bellalta, B., \& Barcelo, J. (2013). Future evolution of CSMA protocols for the IEEE 802.11 standard. IEEE International Conference on Communications Workshops (Vol.38, pp.1274-1279). IEEE.

[11] Bellalta, B., Checco, A., Zocca, A., \& Barcelo, J. (2016). On the interactions between multiple overlapping wlans using channel bonding. IEEE Transactions on Vehicular Technology, 65(2), 796812.

[12] Lee, J., Choi, K. J., \& Kim, K. S. (2016, 19-21 Oct. 2016). Massive MIMO full-duplex for high-efficiency next generation WLAN systems. Paper presented at the 2016 International Conference on Information and Communication Technology Convergence (ICTC).

[13] Qu, Q., Li, B., Yang, M., \& Yan, Z. (2015). FuPlex: A full duplex MAC for the next generation WLAN. Paper presented at the International Conference on Heterogeneous NETWORKING for Quality, Reliability, Security and Robustness.

[14] Agarwal, M., Pasumarthi, D., Biswas, S., \& Nandi, S. (2016). Machine learning approach for detection of flooding DoS attacks in 802.11 networks and attacker localization. International Journal of Machine Learning \& Cybernetics, 7(6), 1035-1051.

[15] Punal, O., Aktas, I., Schnelke, C. J., Abidin, G., Wehrle, K., \& Gross, J. (2014). Machine learning-based jamming detection for IEEE 802.11: Design and experimental evaluation. Paper presented at the World of Wireless, Mobile and Multimedia Networks.

[16] Wang, C., \& Kuo, W. H. (2014). A utility-based resource allocation scheme for IEEE 802.11 WLANs via a machine-learning approach: Springer-Verlag New York, Inc.

[17] Lin, P., \& Lin, T. (2009). Machine-Learning-Based Adaptive Approach for Frame-Size Optimization in Wireless LAN Environments. IEEE Transactions on Vehicular Technology, 58(9), 5060-5073.

[18] Au, E. (2017). IEEE P802.11 - Task Group ay - MEETING UPDATE. Retrieved from http://ieee802.org/11/Reports/tgay_update.htm

[19] McCann, S. (2017). IEEE P802.11 - TASK GROUP AX - GROUP INFORMATION UPDATE. Retrieved from http://grouper.ieee.org/groups/802/11/Reports/tgax_update.html

[20] Y. Liao, K. Bian, L. Song, Z. Han. Full-duplex MAC protocol design and analysis. IEEE Communications Letters 19 (7): 1185-1188, July 2015.

[21] Brown, B. (2017). FAQ: What is 802.11ay wireless technology? Retrieved from http://www.networkworld.com/article/3184827/wi-fi/faq-what-is-80211ay-wireless-

technology.html 